\title{
Erratum to preoperative treatment of anemia-could an ultra- short-term multimodal approach be beneficial for patients undergoing lung surgery?
}

\author{
Sebastian Heinrich, Torsten Loop \\ Department of Anesthesiology and Critical Care, Medical Center-University of Freiburg, Faculty of Medicine, University of Freiburg, Freiburg, \\ Germany \\ Correspondence to: Torsten Loop. Department of Anesthesiology and Critical Care, Medical Center-University of Freiburg, Faculty of Medicine, \\ University of Freiburg, Hugstetter Strasse 55, 79106 Freiburg, Germany. Email: torsten.loop@uniklinik-freiburg.de.
}

doi: $10.21037 /$ jtd.2019.11.24

View this article at: http://dx.doi.org/10.21037/jtd.2019.11.24

Erratum to: J Thorac Dis 2019;11:S1913-5.

Preoperative treatment of anemia — could an ultra-short-term multimodal approach be beneficial for patients undergoing lung surgery?

In the article that appeared on Page 1913-1915, Supplement 15 (September 2019) Issue of the fournal of Thoracic Disease (FTD) (1), there is a mistake on the reference. Here, we sincerely apologize for the mistake.

The reference of the sentence "A recently published meta-analysis including 32 trials with overall 4,750 mixed surgical patients showed a decreased rate of perioperative RBC-transfusion and no increased rate of thromboembolic complications (14)" is currently written below:

14. Spahn DR, Schoenrath F, Spahn GH, et al. Effect of ultra-short-term treatment of patients with iron deficiency or anaemia undergoing cardiac surgery: a prospective randomised trial. Lancet 2019;393:2201-12.

The correct reference should be:

14. Cho BC, Serini J, Zorrilla-Vaca A, et al. Impact of Preoperative Erythropoietin on Allogeneic Blood Transfusions in Surgical Patients: Results From a Systematic Review and Meta-analysis. Anesth Analg 2019;128:981-92.

The authors regret the error.

\section{References}

1. Heinrich S, Loop T. Preoperative treatment of anemia—could an ultra-short-term multimodal approach be beneficial for patients undergoing lung surgery? J Thorac Dis 2019;11:S1913-5.

Cite this article as: Heinrich S, Loop T. Erratum to preoperative treatment of anemia-could an ultra-short-term multimodal approach be beneficial for patients undergoing lung surgery? J Thorac Dis 2020;12(3):1158. doi: 10.21037/ jtd.2019.11.24 